\title{
Nursing assistance to patient with cerebral hemorrhage
}

\author{
Ana Juana Tambasco* \\ Department of Neurosurgery, S. Luca Hospital, Vallo della Lucania, Italy
}

\begin{abstract}
About $25 \%$ of strokes for elderly patients is due to acute cerebral hemorrhage. Consequently, treatments to improve prognosis should be identified. The aim of the present study is to identify a clinical nursing pathway for patients with acute cerebral hemorrhage, improving the clinical effects and nursing satisfaction and reducing adverse reactions.
\end{abstract}

Cerebral hemorrhage is a non-traumatic and spontaneous hemorrhage of cerebral parenchyma, caused by blood vessels rupturing owing to hypertensive arteriolar sclerosis. Cerebral hemorrhage has an acute onset, rapid development, early onset of hemiplegia and disturbance of consciousness. Morbidity and mortality are high, making it one of the most lethal diseases [1]. Acute cerebral hemorrhage has a high incidence in elderly patients, accounting for about $25 \%$ of all strokes [2]. Clinical manifestations following acute cerebral hemorrhage can be brain edema, intracranial hypertension, high fever, cerebral hernia, limb and speech disorder, breathing and heartbeat inhibition, shock and even death have been reported [3]. Cerebral hemorrhage survivors may suffer from a number of complications, which may markedly affect both patients and their families. Cerebral hemorrhage has a high disability rate and even higher mortality rate [4]. In patients with acute cerebral hemorrhage, the provisions that characterize the assistance of the critical neurological patient must be implemented, especially if the state of consciousness is compromised.

\section{State of consciousness}

- Assess the state of consciousness through the Glasgow Coma Scale (GCS) and promptly detect the appearance of new neurological signs

- Monitor pupils frequently (diameter and reactivity to light stimulus)

- In case of psychomotor agitation, reassure the patient and inform the doctor

\section{Breathing}

- Ensure airway patency, keep the patient's head in a slightly raised position, monitor oxygen saturation in the blood

- In case of respiratory depression, position the Guedel cannula, ventilate the patient with a mask and assist the resuscitator in endotracheal intubation maneuvers, if the respiratory autonomy fails

\section{Cardiac activity}

- Frequently detect blood pressure and heart rate

- Position a venous catheter allowing solution infusion and intravenous therapy. The correct dressing of the catheter insertion area will prevent infections

\section{Alteration of the hydroelectrolyte balance}

- Guarantee the patient's hydration via enteral or parenteral

- Keep a daily hydroelectrolyte balance, taking care to monitor hourly diuresis and detect any episodes of polyuria or oliguria

\section{Hygiene}

- Practice total hygiene based on patient autonomy

- Replace the bed linen avoiding sudden movements or rapid changes in position

- Take careful cleaning of the oral cavity even several times a day

\section{Mobilization}

It is necessary to ensure all measures to prevent thrombophlebitis and pressure sores by means of timely interventions. We must therefore:

- Assume correct postures with particular attention to any plegic limbs; the decubitus should be alternated with frequency and the permanence on the hemiplegic side must be shorter than that on the healthy side

- Use anti-bedsores (water, air mattresses, donuts, pillows)

- Passively mobilize the patient

\section{Vomiting}

In case of vomiting it is necessary to ensure a correct airway patency, avoiding the occurrence of "ab ingestis" pneumonia.

- Position the patient in lateral decubitus

- Export any foreign bodies from the oropharynx and aspirate the secretions

- Position a nasogastric tube if the patient is not autonomous

${ }^{\star}$ Correspondence to: Ana Juana Tambasco, Department of Neurosurgery, S. Luca Hospital, Vallo della Lucania, Italy, E-mail: adt2005@tiscali.it

Key words: cerebral hemorrhage, acute cerebral hemorrhage, clinical nursing pathway

Received: November 29, 2018; Accepted: December 04, 2018; Published: December 07, 2018 


\section{Seizures}

- Administer anticonvulsant drugs, according to the prescription

- Evaluate the airway patency and monitor the parameters

- Be ready to transfer the patient to intensive care, where necessary

In the patient with cerebral hemorrhage the nurse will have to monitor the cardiovascular and respiratory parameters and neurological status, putting in place a series of measures to promote improvement and prevent complications.

Patients with subarachnoid or cerebral hemorrhage due to aneurysm or arteriovenous malformation (AVM) are generally considered extremely "delicate" subjects. For such patients, after the acute phase, bed rest is imperative and all the stimuli that can provoke a pressure increase - both psychic (emotions) and physical (noises, trauma in the movements, enema dosage and energetic purgatives, protracted aspirations of tracheal secretions with stimulation of the reflex to cough) - are avoided. It is necessary the exclusion of vascular malformation from the cerebral circulation that can be obtained by surgical clipping (craniotomy) or endovascular treatment with spirals for aneurysms and with craniotomy, endovascular embolization or radiosurgery for AVM.
The post-operative course of the patient provides a close control of the level of consciousness, the presence of headache or neck stiffness and the possible emergence of post-intervention neurological deficits. Intensive control of the blood pressure values is necessary to avoid ischemic complications due to vasospasm. We must not forget the possibility of the emergence of obstructive hydrocephalus. In addition, patients treated with endovascular procedure are often kept on anticoagulate with heparin for one week and on antiplatelet therapy for three months: they therefore require frequent coagulation checks, especially in the first postoperative week.

\section{References}

1. Manning LS, Robinson TG (2015) New insights into blood pressure control for intracerebral hemorrhage. Front Neurol Neurosci 7: 35-50 [Crossref]

2. Sato S, Carcel C, Anderson CS (2015) Blood pressure management after intracerebral hemorrhage. Curr Treat Options Neurol 17: 49 [Crossref]

3. Yousef KM, Balzer JR, Bender CM, Hoffman LA, Poloyac SM, et al. (2015) Cerebral perfusion pressure and delayed cerebral ischemia after aneurysmal subarachnoid hemorrhage. Am J Crit Care 24: e65-e71. [Crossref]

4. Christensen MC, Mayer S, Ferran JM (2009) Quality of life after intracerebral hemorrhage: results of the Factor Seven for Acute Hemorrhagic Stroke (FAST) trial. Stroke 40: 1677-1682. [Crossref]

Copyright: (C2018 Tambasco AJ. This is an open-access article distributed under the terms of the Creative Commons Attribution License, which permits unrestricted use, distribution, and reproduction in any medium, provided the original author and source are credited. 